\title{
Los estudiantes de secundaria y el vínculo comunitario. Un estudio en tres instituciones educativas de secundaria en Colombia*
}

\author{
Nancy Palacios Mena ${ }^{1}$ \\ (iD http://orcid.org/0000-0001-5519-7515 \\ Universidad del Tolima, Colombia
}

DOI: http://dx.doi.org/10.17081/eduhum.19.32.2532

Recibido: 12 de junio de 2016

Aceptado:15 de septiembre de 2016

\section{High school students and relationship with the community. A study in three secondary educational institutions in Colombia}

Palabras clave:

Socialización juvenil,

Socialización de jóvenes escolares,

Relación juventud-comunidad.

\begin{abstract}
Resumen
Este artículo es producto de una investigación que tuvo como objetivo principal analizar procesos de socialización juvenil en la escuela y fuera de ella. El texto se ocupa de la vinculación de jóvenes a instancias de la vida comunitaria y de sus percepciones frente a problemáticas que aquejan sus entornos inmediatos. Se utilizó un diseño metodológico mixto, que combinó datos cuantitativos (una encuesta) y cualitativos (entrevistas y análisis documental). Concluimos que la vinculación o no de los jóvenes a proyectos o programas de las comunidades depende de las dinámicas que se dan en su interior, de las percepciones que logran construir de dichas dinámicas y de la manera como los adultos los involucran y crean o no oportunidades para el agenciamiento juvenil en dichos proyectos.
\end{abstract}

\begin{abstract}
The article is the result of an investigation that had as main objective to analyze youth socialization processes at school and beyond. The text deals with the field of socialization that involves linking youth at the instances of community life and their perceptions of the problems facing their immediate surroundings. A mixed methodological design that combined quantitative data (survey) and qualitative (interviews and document analysis) was used. We conclude that the participation or not of the youth to projects or community programs depend on the dynamics that occur within them, perceptions that the youth construct out of these dynamics and how adults involved and create or not opportunities for youth participation in these projects.
\end{abstract}

Juvenile socialization,

Socialization of schoolchildren, Youth community relationship.

\section{(c) $\underset{\mathrm{BY}}{(\mathrm{NC}} \bigodot_{\mathrm{ND}}$}

Referencia de este artículo (APA): Palacios, N. (2017). Los estudiantes de secundaria y el vínculo comunitario. Un estudio en tres instituciones educativas de secundaria en Colombia. En Revista Educación y Humanismo, 19(32), 67-87. http://dx.doi.org/10.17081/ eduhum.19.32.2532

\footnotetext{
Este artículo se deriva de la investigación titulada "Derechos, subjetividad y socialización política en la escuela. un estudio en tres instituciones educativas de secundaria”. Tesis con la que se optó por el título de Doctora en Ciencias Sociales, Niñez y Juventud. La tesis recibió la Mención de Honor Magna cum laude.

1. Doctora en Ciencias Sociales, Niñez y Juventud, CINDE-Universidad de Manizales. Licenciada en Ciencias Sociales y Magíster en Sociología, Universidad del Valle. Profesora de la Universidad del Tolima, Colombia npalaciosme@ut.edu.co
} 


\section{Introducción}

Uno de los principales retos de la escuela secundaria actual consiste en comprender quiénes son los jóvenes que asisten a las aulas, es decir, saber qué piensan, qué los emociona, qué los mueve, qué los alegra, qué los entristece, qué los motiva a estar en colegio o a no querer permanecer en él. La incomprensión de estos aspectos nos ha llevado a centrar la preocupación en los efectos de los comportamientos juveniles y sus consecuencias en la vida escolar, familiar o la relación con los pares. De lo anterior se deriva una gama de estudios interesados en explicar el matoneo escolar y "el mal comportamiento" que impide que los alumnos aprendan, pero que además provoca rivalidades entre los jóvenes, y entre estos con los docentes y directivos.

Consideramos al respecto que si bien es valioso caracterizar los comportamientos de los jóvenes y sus efectos en diferentes contextos, también es importante determinar cómo se construyen las subjetividades juveniles y qué papel juegan los diferentes ámbitos de socialización en dicha construcción. Más en concreto, es clave desentrañar los factores asociados a los procesos de configuración de las subjetividades de los jóvenes escolares en función de los escenarios de socialización, porque creemos que dicho desentrañamiento nos dará algunas luces para comprender los discursos y las prácticas juveniles, lo que, a su vez, permitiría a la escuela emprender acciones pedagógicas y didácticas para evitar la violencia, el matoneo, la deserción escolar y los conflictos disciplinarios, Se entiende el conflicto disciplinario como el conjunto de tensiones, desacuerdos y discrepancias que se presentan entre los estudiantes, docentes y directivos, en torno al concepto, la importancia y los mecanismos utilizados para concretar la disciplina en las instituciones educativas (Palacios, 2008). Si logramos entender cómo se construyen los jóvenes estudiantes en los diferentes contextos de socialización, muy posiblemente se podrá pensar e implementar acciones pedagógicas desde la escuela, que orienten las prácticas juveniles que afectan sus aprendizajes y la convivencia escolar. En este artículo, nos ocuparemos de una parte de la socialización juvenil: la relacionada con los vínculos de los jóvenes en instancias de la vida comunitaria y sus percepciones frente a las problemáticas que aquejan sus entornos inmediatos. Tradicionalmente, se les ha tildado de apolíticos y poco preocupados por vincularse activamente a procesos de participación comunitaria, pero, en este trabajo, partimos de una intuición que nos indica que su vinculación o no a proyectos o programas de las comunidades depende de las dinámicas que se dan en su interior, de las percepciones que tienen sobre dichas dinámicas y de la manera como los adultos los involucran y crean o no oportunidades para el agenciamiento juvenil en dichos procesos.

\section{Método}

En la investigación que dio origen al artículo se utilizó un diseño metodológico mixto. Este tipo de métodos agrega complejidad al diseño de una investigación y contempla las ventajas de 
cada uno de los enfoques, dado que se trata de un proceso en el que se recolectan, analizan y vinculan datos cuantitativos y cualitativos en un mismo estudio.

La utilización de metodologías mixtas supone la superación de visiones únicas sobre un ambiente particular (enfoque cualitativo), y que los resultados sean una simple instancia en el marco de algún conjunto de principios generalizados (enfoque cuantitativo) (Hernández, Fernández \& Baptista, 2003). En este sentido, se rescata la importancia del pluralismo metodológico, por cuanto los métodos mixtos representan un intento por legitimar la utilización de múltiples enfoques para resolver los problemas de investigación y no limitan las elecciones de los investigadores. La utilización de los métodos mixtos supone, así, una forma creativa, expansiva, plural y complementaria de dirigir los estudios, en el entendimiento de que, en el proceso investigativo, el método debe ser adecuado al planteamiento del problema, para que responda a los interrogantes propuestos de la manea más útil, profunda y completa.

Así, con los diseños metodológicos mixtos se logra obtener una mayor variedad de perspectivas del problema, frecuencia, magnitud, amplitud, profundidad, complejidad, generalización y comprensión; en palabras de Castro y Molinari (2009), la combinación de las técnicas cualitativas y cuantitativas ayuda a clarificar y a formular el planteamiento del problema, así como las formas más apropiadas de estudiar y priorizar los problemas de investigación.
La particularidad de un estudio mixto radica, consecuentemente, en recolectar y analizar datos cuantitativos y cualitativos, de tal manera que los hallazgos sean iluminadores. Para ello se requiere una verdadera integración de la información obtenida y su integración de manera significativa, pues, el análisis, más que la suma de los datos cualitativos y cuantitativos, es un reto de legitimación que solo se costea si se obtienen resultados creíbles, confiables, transferibles, y con posibilidad de confirmación. Esto sin perder de vista que tanto el componente cualitativo como el cuantitativo introducen sus propios problemas de representatividad y legitimidad (Hernández et al., 2003).

\section{Participantes}

El universo empírico de la investigación fue constituido por tres establecimientos educativos de secundaria, públicos, mixtos y ubicados en la zona urbana de tres municipios: Ansermanuevo, Cartago y Pereira. Los estudiantes participantes pertenecen a los estratos uno, dos y tres, excepto algunos casos del estrato cuatro y cinco en las instituciones de Cartago y Pereira.

\section{Variables}

El estudio que dio origen a este artículo comprende tres grandes categorías: la socialización, los derechos y la subjetividad en la escuela. En este trabajo nos ocupamos de una parte de esa socialización, la relacionada con el vínculo de los jóvenes estudiantes con asociaciones comunitarias y la percepción que estos tienen sobre los problemas sociales y sobre 
el impacto de dichos problemas en su vida. Al respecto, se incluyeron variables como: pertenencia y participación en agrupaciones u organizaciones comunitarias o barriales, acciones realizadas en agrupaciones $\mathrm{u}$ organizaciones, influencia de los problemas sociales en la vida de los jóvenes estudiantes, reflexiones que generan problemáticas juveniles. La inclusión de estas variables obedece a la intención de analizar $\mathrm{su}$ vinculación con las organizaciones de los contextos en los cuales interactúan, para determinar si la vinculación influye en las posturas políticas que dichos jóvenes expresan. La inclusión de las percepciones de los problemas sociales apunta a determinar de qué manera la preocupación por sus problemáticas los interesa o no por participar en alguna organización, pues, se pretende establecer si ellos ven en las respectivas organizaciones instancias efectivas para tramitar las problemáticas comunitarias.

Instrumentos de recolección de información

Se aplicó un cuestionario para recoger información cuantitativa sobre las variables descritas anteriormente. Debido a la diferencia en número de alumnos de los colegios participantes, se requirió un diseño de la muestra en dos etapas (Tabla 1): un muestreo estratificado proporcional y un muestreo aleatorio sistemático. Con el muestreo estratificado proporcional, se garantizó un reparto equitativo, de manera que cada colegio tuviera la misma proporción de estudiantes participantes según su número total de alumnos. Por su parte, el muestreo aleatorio sistemático garantizó que cualquiera de los estudiantes de cada una de las instituciones estrato- pudiera hacer parte de la muestra, con otras palabras, fue la garantía de que el muestreo se hiciera equitativamente y de manera aleatoria sobre la totalidad de la población.

Tabla 1. Definición de la muestra

\begin{tabular}{|l|l|}
\hline $\begin{array}{c}\text { Determinación del tamaño } \\
\text { muestral para la proporción }\end{array}$ & \multicolumn{1}{|c|}{ Fórmula } \\
\hline Tamaño de la población & $\mathrm{N}=4654$ \\
\hline $\begin{array}{l}\text { Nivel de confianza } \\
\text { recomendada para estudios } \\
\text { sociales }\end{array}$ & $1-\propto=95 \% \mathrm{Z}=1,96$ \\
\hline $\begin{array}{l}\text { Proporción de la población } \\
\text { con la característica estudiada }\end{array}$ & $\begin{array}{l}\mathrm{p}=\mathrm{q}=0,5 \\
\text { Fórmula } \\
=355 \text { unidades muestrales }\end{array}$ \\
\hline
\end{tabular}

Fuente: Elaboración propia

Después de determinar el tamaño muestral para cada estrato, se realizó muestreo aleatorio sistemático en cada institución, debido a que se tenía claramente identificado cada uno de los elementos pertenecientes a cada estrato (ver fórmula aplicada para el caso del ejemplo del Colegio 1). Para esto se generaron números aleatorios con la ayuda de un generador de números aleatorios digital. Ejemplo:

\section{Colegio 1}

Intervalo de selección $=\mathrm{N} / \mathrm{n}=1500 / 110=$ $13,63 \approx 14$

Número de arranque $=$ aleatorio.entre $(1,14)=$ 10

Individuos seleccionados: 10, 24, 38, 52, 66, $80,94,108,122 \ldots \ldots \ldots \ldots$ 
También se utilizaron programas estadísticos para calcular muestras de manera computarizada, tanto para la prueba piloto como para la encuesta definitiva (Tabla 2). En este caso, el margen de error de la muestra fue del $15 \%$.

Tabla 2. Definición de la muestra del cuestionario

\begin{tabular}{|l|c|c|c|c|}
\hline Estratos & $\begin{array}{c}\text { Población } \\
\text { total }\end{array}$ & $\begin{array}{c}\text { Número } \\
\text { de encuestas } \\
\text { proyectadas } \\
\text { en cada } \\
\text { estrato }\end{array}$ & $\begin{array}{c}\text { Número } \\
\text { de encuestas } \\
\text { aplicadas } \\
\text { en cada } \\
\text { estrato }\end{array}$ & $\begin{array}{c}\text { Número } \\
\text { de encuestas } \\
\text { proyectadas } \\
\text { para la } \\
\text { prueba piloto }\end{array}$ \\
\hline Colegio 1 & 1502 & 110 & 76 & 31 \\
\hline Colegio 2 & 2168 & 160 & 132 & 45 \\
\hline Colegio 3 & 986 & 74 & 79 & 21 \\
\hline Total & 4654 & 344 & 287 & 97 \\
\hline
\end{tabular}

Fuente: Elaboración propia

Para comprobar la validez y confiabilidad del cuestionario, se ejecutaron dos acciones: primero se aplicó una prueba piloto del instrumento con un tamaño de muestra calculado con el mismo programa de computación con el que se definió el tamaño de la muestra para la aplicación del instrumento definitivo; luego el cuestionario se sometió a evaluación de un experto. El tamaño total de la muestra para la prueba piloto del cuestionario fue de 97 (Ver Tabla 1, columna 5). Cabe aclarar que la aplicación de la prueba piloto nos permitió ajustar y reformar el instrumento inicial, entre los ajustes más significativos que se realizaron tras su aplicación se pueden destacar: la reducción de preguntas abiertas, el cambio en las opciones de respuestas a algunas de las preguntas porque generaban ambigüedad a la hora de contestarlas, el reemplazo de algunos términos que los estudiantes no entendían con facilidad, la reducción del tamaño general del cuestionario, la reformulación de algunas preguntas que provocaron confusión en los estudiantes, la inclusión de preguntas que los estudiantes consideraron importantes. En conclusión, el ejercicio de pilotaje fue muy útil para consolidar y corregir el cuestionario que finalmente se aplicó.

Como ya se ha dicho, con posterioridad a las modificaciones del cuestionario resultado de la aplicación de la prueba piloto y con el objetivo de mejorar más el instrumento, este también fue sometido a la evaluación de un experto en educación y en la aplicación de encuestas. Dicha evaluación fue de gran utilidad, no solo porque permitió conocer la lectura del cuestionario de una persona externa, sino porque las observaciones realizadas ayudaron a afinarlo y a reorientar algunas preguntas planteadas inicialmente. El evaluador señaló que algunas preguntas podían generar confusión o requerir explicación por parte de los estudiantes; en consecuencia, sugirió tener en cuenta que las preguntas apuntaran a la percepción sobre dinámicas escolares en un nivel de abstracción que debía ser consecuente con la etapa del desarrollo en la que se encontraban los alumnos; en algún caso, además, evidenció que no se identificaba con claridad el elemento de la categoría al cual correspondía la pregunta según la definición dada.

Por otra parte, en la recolección de datos cualitativos se realizaron 15 entrevistas semiestructuradas en las cuales se indagó sobre espacios 
de socialización como casa, colegio, barrio; las formas de relación que se establecen en dichos contextos de socialización, la influencia de familiares, pares, vecinos en la socialización, la pertenencia a grupos estudiantiles, comunitarios, deportivos; las actividades que se realizan en el contexto escolar y por fuera de él, y el tiempo dedicado a las mismas. Debido a que el estudio que da origen al artículo tuvo como unidad de análisis tres categorías: los derechos, la socialización y la subjetividad política, los estudiantes entrevistados fueron los que han participado activamente en procesos de reivindicación de derechos, y líderes estudiantiles como el personero o el contralor del colegio. El contenido del texto de la entrevista buscaba principalmente que los estudiantes relataran eventos de reivindicación de derechos en los que hubieran participado o que hubieran conocido, $\mathrm{y}$ contaran algunos aspectos de su vida cotidiana, como la relación con sus amigos, las vivencias familiares, sus actividades recreativas, los espacios y las interacciones con los compañeros del colegio, así como las relaciones y vínculos en sus contextos comunitarios.

Una última técnica utilizada en este trabajo de investigación fue el análisis documental; en este sentido, se revisó una gran variedad de documentos de acciones de reivindicación de derechos como actas de reunión, tutelas, derechos de petición, quejas, apelaciones, solicitudes de veeduría y acompañamiento a estudiantes.

\section{Análisis de los datos}

Para sistematizar los datos cuantitativos se elaboraron tablas de contingencia y diagramas de barras apiladas. Estas gráficas estadísticas se utilizan para comparar el porcentaje que contribuye a cada valor en un total, a través de categorías que se representan mediante rectángulos horizontales. La presentación de una u otra depende del número de categorías que contenga, con el fin de cuidar el componente estético del documento.

La sistematización de las entrevistas requirió de dos etapas, la primera fue de transcripción y la segunda de tematización. En la primera, se escribió literalmente el contenido de cada una de las 15 entrevistas y en la segunda, se elaboró una matriz con fragmentos de las respuestas. Esta tarea de sistematización requirió una labor de etiqueta y codificación de los fragmentos en función de las variables que ya se han indicado (Tabla 3). El procedimiento para el análisis de la información de la entrevista fue el siguiente: una vez se transcribió cada una, se realizó una primera lectura y se subrayaron con colores los temas generales tocados por los estudiantes. En un segundo momento, se sacaron los fragmentos ya señalados y se construyó con ellos una matriz, utilizando colores y códigos, como se muestra en la Tabla 3:

El análisis de los documentos fue de utilidad 
Tabla 3. Modelo de sistematización de los datos cualitativos. Definición de la muestra

\begin{tabular}{|c|c|c|c|c|}
\hline$N^{\circ}$ de entrevista & Categoría & Código & Sub-categoría & Fragmento \\
\hline 7 & Socialización & $\begin{array}{c}\text { Socialización } \\
\text { Escolar }\end{array}$ & S.1 & $\begin{array}{l}\text { El muro está desde que yo llegué al colegio, es un lugar donde } \\
\text { uno puede escribir lo que quiera siempre y cuando no ofenda ni } \\
\text { irrespete a nadie, allí también uno se hace charlar y pasar el rato. }\end{array}$ \\
\hline
\end{tabular}

Fuente: Elaboración propia

para establecer la manera en que los actores escolares usan y recrean los derechos, la forma cómo funcionan estos y las dinámicas sociales que han generado tanto la implementación como la reivindicación de dichos derechos. La lectura de los documentos fue registrada en tarjetas o fichas de trabajo, que consignaban la información básica de cada uno: título, carácter del documento, denominación, autor y destinatario, origen, periodicidad (si la tiene), procedencia y fecha de producción, y contenido (De la Torre \& Navarro, 1990). Estos datos fueron muy útiles en la investigación para conocer las motivaciones del autor y las situaciones en que se produjeron.

\section{Resultados}

\section{Percepciones e influencias de la vida fuera}

\section{del colegio}

Como ya se ha planteado, en este artículo

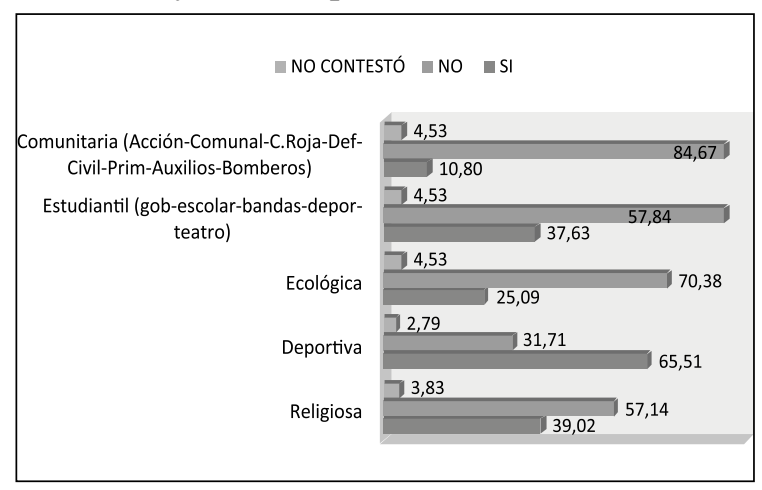

Gráfica 1. Participación en agrupaciones

Fuente: Elaboración propia se analiza la forma como los estudiantes dicen relacionarse con la comunidad de la que hacen parte. En concreto, se indagó la vinculación de los jóvenes estudiantes a grupos comunitarios; al respecto, el 65,5\% afirmó participar en grupos deportivos, el $39 \%$ a grupos religiosos, el $25 \%$ a grupos ecológicos y el 10,8 \% a agrupaciones comunitarias como los Bomberos, la Defensa Civil, la Cruz Roja y las Juntas de Acción Comunal. Los resultados aparecen en la Gráfica 1.

La información anterior, fue complementada en las entrevistas. Se registran las respuestas en la Tabla 4.

De las entrevistas podemos inferir que el fútbol y el microfútbol son actividades que unen a los jóvenes en agrupaciones comunitarias. Muy seguramente la pertenencia a estos equipos implica para los estudiantes un espacio de socialización complementario al del colegio, al que no solo le dedican parte de su tiempo libre, sino que también han establecido con ellos vínculos afectivos importantes.

Los testimonios de los estudiantes reflejados en las entrevistas dejan ver, además, que contrario al entusiasmo que genera la inclusión 
Tabla 4. Fragmentos de las entrevistas contestando a la pregunta por la pertenencia a organizaciones comunitarias

\begin{tabular}{|l|l|}
\hline \multirow{5}{*}{ ¿Participas de alguna agrupación } & $\begin{array}{l}\text { Yo no participo en agrupaciones, porque solo se reúnen para hablar de política en tiempo de } \\
\text { elecciones, el resto del año, no hablan de la basura que la gente deja en la esquina, de la calle que } \\
\text { tiene huecos, de la inseguridad del barrio, esa junta no sirve para nada. Entrevista: Estudiante del } \\
\text { colegio de Pereira }\end{array}$ \\
\cline { 2 - 3 } & $\begin{array}{l}\text { Participo en el equipo de fútbol del barrio, es chévere, vamos a jugar a otros barrios, a veces fuera } \\
\text { del municipio. El equipo tiene uniformes y ha recibido el apoyo de los comerciantes de por acá cerca, } \\
\text { para qué, es bueno el equipo. Entrevista: Estudiante del colegio de Cartago }\end{array}$ \\
\cline { 2 - 3 } & $\begin{array}{l}\text { Yo sí he participado en el equipo micro de uno de los supermercados, hemos estado en finales y } \\
\text { todo, participamos en el torneo de micro que hicieron en el parque, estuvo bueno, a veces jugamos } \\
\text { en el estadio por las noches contra otros equipos, o bajamos a jugar a gramalote o arriba del poli. } \\
\text { Entrevista: Estudiante del colegio de Ansermanuevo }\end{array}$ \\
\cline { 2 - 3 } & $\begin{array}{l}\text { Yo no participo en acción comunal, allí van solo adultos y viejitos. Yo nunca he visto gente joven que } \\
\text { vaya a esas reuniones, sería bueno ir porque el barrio sí tiene mucha necesidad para qué y en cuanto } \\
\text { a grupo ecológico, en el barrio no hay, el único que conozco en el pueblo es del colegio, no más. } \\
\text { Entrevista Estudiante del colegio de Ansermanuevo }\end{array}$ \\
& $\begin{array}{l}\text { Yo no he participado, pero ahora que pregunta sería hasta bueno, mi abuela sí ha ido a votar y a } \\
\text { reuniones, pero luego viene aburrida porque dice que los que ganan ya no piensan en el barrio, sino } \\
\text { que se unen a los políticos y buscan un puesto para ellos o para sus hijos en la alcaldía, se quedan } \\
\text { con las cosas que mandan para la comunidad. Los jóvenes sí debiéramos ponernos pilas con eso. } \\
\text { Entrevista: Estudiante colegio de Pereira }\end{array}$ \\
\hline
\end{tabular}

Fuente: Elaboración propia

en agrupaciones deportivas, la idea de participar en Juntas de Acción Comunal no los motiva, pues estos son vistos como espacios para adultos y de la tercera edad, "viejitos". Asimismo, los jóvenes perciben que quienes se vinculan a estos grupos solo buscan intereses personales para lograr favores políticos y no el bienestar de la comunidad, perciben también alianzas entre quienes se convierten en líderes comunitarios y politiqueros para ejecutar acciones de corrupción a cambio de votos. Podemos decir entonces que en las comunidades no se ha explotado el potencial de los jóvenes en agrupaciones comunitarias. Según lo expresado por los estudiantes aparte de que no han sido convocados, tampoco se han puesto en práctica estrategias que inciten a los jóvenes a vincularse y que los concienticen de la importancia y la necesidad de su dinamismo, sus ideas y sus iniciativas dentro de estos espacios.

Sin embargo, si bien los datos recogidos no muestran una fuerte participación de los estudiantes en asociaciones y actividades comunitarias, hay un estudiante que menciona su participación en el grupo de los scouts para demostrar los valores que tiene y con ello quitarle peso a las acusaciones de la institución de ser un alumno indisciplinado que no cumple las normas (Tabla 5).

El caso del anterior estudiante sugiere que él es consciente del reconocimiento y el valor que tiene esta agrupación para la comunidad, considera que su participación no es solo un 
Tabla 5. Estructura del derecho de petición de un estudiante que solicita que su participación en un grupo sea un atenuante ante una falta disciplinaria en el colegio

Tiene por objeto la acción de restauración de mis derechos fundamentales. Al debido proceso, a la educación, que han sido vulnerados al expulsarme indebidamente de la institución educativa en la cual es el rector

\begin{tabular}{|l|l|}
\hline Hechos & $\begin{array}{l}\text { Soy estudiante del grado 7: } 5 \text { donde he cursado mis estudios de bachillerato desde sexto grado. Tengo quince años y soy } \\
\text { miembro activo de la organización scout (Quimbayas 411 de la ciudad de Cartago) } \\
\text { Deseo estudiar y culminar en la institución porque allí es donde he podido superarme como persona y estudiante. Tengo } \\
\text { mis compañeros scouts y de los grupos ecológicos a los cuales pertenezco }\end{array}$ \\
\hline Peticiones & $\begin{array}{l}\text { Se me tutelen mis derechos al debido proceso, a la defensa y a la educación. Conforme lo plantea el manual de convivencia. } \\
\text { El señor rector no realizó la notificación a mi director de grupo y al coordinador. Tampoco ha sido convocado el comité } \\
\text { institucional de convivencia, organismo que funciona en la institución educativa y que es competente para realizar } \\
\text { las correcciones pertinentes. En mi caso no se dio una sanción formativa y pedagógica, el señor rector solo dio una } \\
\text { demostración de poder y me expulsa fulminantemente. El manual de convivencia califica las faltas cometidas por nosotros } \\
\text { los estudiantes en leves y graves (el rector nos agrupó como si todos hubiéramos cometido una falta) y dio un fallo en } \\
\text { grupo, no hizo un análisis individual } \\
\text { Deseo volver a estudiar porque es mi proyecto inmediato de vida, en este momento estoy por fuera de la institución y dada } \\
\text { mi condición de adolescente la calle ofrece muchos peligros. No deseo ser un colombiano en situación de calle }\end{array}$ \\
\hline Derechos & $\begin{array}{l}\text { Son fundamentos de derecho de mi petición consagrados en el artículo No } 29: \text { debido proceso y de defensa En concordancia } \\
\text { con el derecho a la educación } \\
\text { Fragmento de tutela solicitando el debido proceso por expulsión de la institución educativa (Cartago) }\end{array}$ \\
\hline
\end{tabular}

atenuante a su falta disciplinaria sino que dicha pertenencia habla bien de él y de sus valores.

En la Tabla 6 se presentan las respuestas de lación a grupos y actividades de la vida comunitaria:

los estudiantes al interrogante sobre su vincu-

Tabla 6. Participación en actividades comunitarias

\begin{tabular}{|l|c|c|c|c|c|}
\hline $\begin{array}{l}\text { 20. SS18. Como joven ¿En cuál de las siguientes actividades de } \\
\text { tu barrio participas? }\end{array}$ & Nunca & Algunas veces & Casi siempre & Siempre & No Contestó \\
\hline Jornadas de limpieza y embellecimiento & 54,01 & 22,30 & 12,20 & 8,36 & 3,14 \\
\hline Elección de la Junta de Acción Comunal & 62,37 & 16,72 & 13,24 & 4,88 & 2,79 \\
\hline Campañas políticas en tiempos de elección & 65,51 & 15,33 & 10,45 & 5,57 & 3,14 \\
\hline Protesta reclamando necesidades del barrio & 49,13 & 18,47 & 17,77 & 10,45 & 4,18 \\
\hline Celebraciones & 36,93 & 19,51 & 25,09 & 14,63 & 3,83 \\
\hline Actividades de recolección de dinero para el barrio & 40,07 & 24,04 & 19,16 & 12,20 & 4,53 \\
\hline Actos de solidaridad con algún vecino & 25,78 & 14,29 & 24,39 & 9,41 & 26,13 \\
\hline
\end{tabular}

Fuente: Elaboración propia 
las que un mayor número de estudiantes dice integrarse son las celebraciones y la recolección de dinero para el barrio. En la primera, un 14,6 $\%$ afirmó que siempre participa y el $25 \%$ dijo que casi siempre. En la segunda, la repartición en ambas alternativas es del 12,2\% y el 19,1 $\%$, respectivamente. Se resalta que el $24,3 \%$ asegura que casi siempre participa en actos de solidaridad con vecinos y el $17,7 \%$ dice que casi siempre ha protestado por las necesidades del barrio.

Con el objetivo de determinar el impacto que les causan, también se preguntó a los estudiantes: “QQué influencia tienen sus actitudes y comportamientos en algunas problemáticas sociales?"

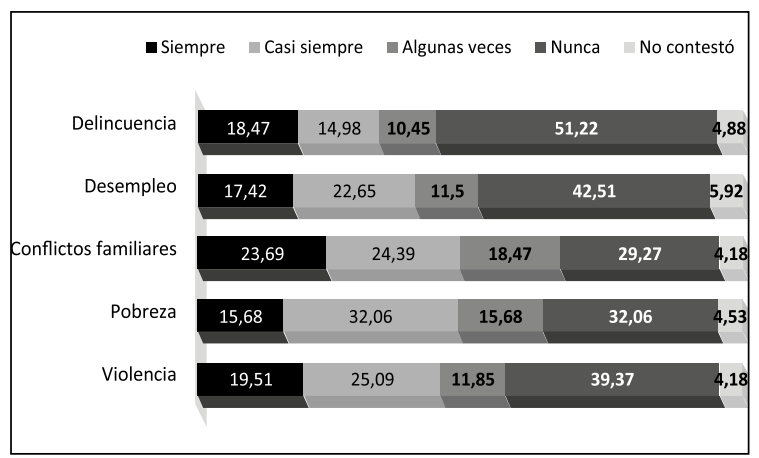

Gráfica 2. Influencias de problemáticas sociales Fuente: Elaboración propia

(Gráfica 2):

La suma de las opciones "Siempre" y "Casi siempre" indica que lo que más influye en los estudiantes son los conflictos familiares, con 42 , $1 \%$. Los siguen la violencia y la pobreza con el $31,3 \%$. Un poco menos de influencia ejercen el desempleo y la delincuencia con $28,9 \%$.
En general, la relativamente baja participación de los estudiantes en las actividades y grupos de sus comunidades preocupa por el gran valor que pueden tener las organizaciones comunitarias en sociedades como la nuestra: pueden, por ejemplo, gestionar recursos, pero además ser veedoras y fiscalizadoras de irregularidades en su uso y ejecución. Pero si, como expresan los datos, la principal actividad en la que los jóvenes se acercan a la comunidad consiste en recoger dinero para celebraciones, esta posibilidad es lejana. Se debe tener en cuenta que en realidad muchas organizaciones barriales y vecinales terminan salpicadas por la corrupción y la politiquería, lo que causa pérdida de credibilidad y desmotivación para hacer parte de ellas. Desde la perspectiva de Alvarado y Ospina (2007), el efecto de situaciones como esta se refleja en la alta frustración, desconfianza e impotencia que sienten las personas al no encontrar poderes claros que orienten la vida en común; según estos autores la banalización de la política incide en un repliegue de los espacios públicos, en un desplazamiento al ámbito de lo privado e individual, y en una reducción del ámbito de lo sociocultural.

Para Tanaka (1995), la no expresión política del protagonismo juvenil a nivel social no tiene por qué entenderse necesariamente como despolitización o desinterés de los jóvenes al respecto; esta autora considera que su ausencia de participación, para algunos espacios y actividades, se explica más por los costos que implica la acción colectiva, que por ausencia de interés. Desde este 
punto de vista, su no vinculación a dinámicas comunitarias es consecuencia de un fenómeno más estructural, difícilmente puede preverse su protagonismo en tanto actores sociales organizados, dadas las formas de socialización que parecen gestarse en la reestructuración neoliberal actualmente en curso.

Con relación a esto último, en la discusión y conclusiones de su tesis doctoral, sobre las organizaciones sociales juveniles, Delgado (2005) destaca que, frente a los marcos de injusticia, estas organizaciones hacen diversas lecturas de sus contextos. Estas percepciones de su realidad se asumen como paradojas y centran su atención en tres lugares esenciales que demarcan un futuro incierto: "en primer lugar, se esboza la contradicción existente entre el crecimiento económico y la inequidad en la distribución del ingreso; en segunda instancia enuncian su preocupación por el marcado avance tecnológico de los últimos tiempos y la inequidad en la distribución del conocimiento, y finalmente, alcanzan a plantear el contrasentido entre el auge de la democracia y el debilitamiento de lo político" (p.131). En este orden de ideas, el mismo autor afirma que los y las jóvenes identifican una serie de situaciones asociadas a su vida y a sus comunidades y, dentro de ellas, las problemáticas más acuciantes son el desempleo, el subempleo, la pobreza y la marginalidad social, los diversos tipos de violencia. También denuncian su escepticismo ante las prácticas políticas de sus autoridades y líderes comunitarios.
Según Garcés (2010), las formas de agrupación juvenil promovidas por el mundo adulto tienden más a constituirse en organizaciones estructuradas con personería jurídica y con intereses y acciones precisas a corto y largo plazo; en contraste, otras formas de agrupación juvenil se caracterizan por intereses sociopolíticos alternativos; por ejemplo, se resisten a la organización jerárquica adultocéntrica y prefieren el gobierno horizontal, la autogestión y abogan por la culturización de la política, así como por acciones plurales directas. Vemos entonces que los colectivos juveniles se crean en razón de la defensa de derechos asociados a aspectos sociales: edad, género, orientación sexual, y cuentan con dinámicas de agrupación y de acción que se alejan de las estructuras formales de tipo ideológico y partidista. Los intereses y escenarios de los colectivos juveniles se hallan centrados en la cultura, el reconocimiento de las identidades diversas, los derechos humanos, entre otros aspectos. En este orden de ideas, es evidente entonces que los intereses de los jóvenes están alejados de los intereses que tradicionalmente han reivindicado las organizaciones comunitarias.

Ahora los jóvenes privilegian el participar en "redes informales", construidas para fines concretos e inmediatos, más que en organizaciones formales y fuertemente estructuradas. Estas renovadas formas de agrupación juvenil son nombradas por algunos investigadores como "nuevos movimientos sociales" (Garcés, 2010). 
Los referentes que los congregan están más cerca del arte, la música, la cultura, el deporte $\mathrm{y}$, en general, del universo sensible que de los "grandes ideales de transformación social".

El citado autor concluye que dichos referentes no solo operan como ejes medulares en la construcción de la identidad juvenil, lo que ya es un hecho político, sino que, a su entender, presentan una dimensión política en distintos sentidos: primero, porque apuntan a la visibilidad del sujeto joven y la búsqueda de reconocimiento y legitimación social de sus prácticas; segundo, porque entran en la escena del "mercado político" para lograr que sus proyectos sean considerados en los presupuestos oficiales; y tercero, porque toman distancia o incluso se convierten en grandes cuestionadores desde la disidencia o la resistencia de las prácticas políticas hegemónicas.

Los autores como Garcés insisten en que la formación ciudadana, es decir, la socialización política de los jóvenes, requiere que se incentive en ellos la acción política desde la escuela, entendida como la capacidad de participar en una construcción social (Lachner, 1999, citada por Alvarado \& Ospina, 2007). También se sugiere que la formación en ciudadanía guarde relación con el vínculo social; que se le apueste a una clara adhesión a la democracia, es decir, que se base en procesos organizados y colectivos de confianza social y de reciprocidad; que recoja dimensiones del ámbito privado, pero que no se reduzca a él; “el énfasis en la participación en organizaciones sociales, es decir la socialización política, como práctica social, solo puede darse en el ámbito de la colectividad y de los intereses sociales" (Vasco, Quintero \& Echavarría, 2007, p.96). Este grupo de investigadores plantea la importancia de trabajar de manera sistemática para ampliar el círculo ético en la educación de los jóvenes, ya que su mirada de lo cotidiano y lo inmediato no implica su comprensión de las exigencias éticas, pues estas no se agotan allí; de no ser así, corren el riesgo de excluir y discriminar a personas y grupos que no se identifican con el círculo inmediato y se diferencian en forma importante de él.

Bandit (1999) sostiene que las asociaciones que más le interesan a los jóvenes son las especializadas en organizar actividades recreativas y de ocio, fundamentalmente en el plano del deporte y, en menor medida, en el plano cultural. En consecuencia, el tipo de participación predominante es estrictamente funcional. Los jóvenes participan de manera selectiva en aquellas actividades de su asociación y se relacionan con ella a la manera en que lo hacen "clientes" cuando seleccionan una oferta de servicios. En todo caso, según el mismo Bandit (1999), la implementación de la participación juvenil en la vida social y política de la sociedad exige también a los "adultos" la superación de la visión predominante de la subjetividad juvenil de una manera infantili- 
zante, es decir, el dejar de ver a los jóvenes como seres dependientes e inmaduros que requieren de dirección y liderazgo en el sentido negativo de la palabra, para no ponerse en peligro a sí mismos y a los demás. Por el contrario, se trata de aceptar a los jóvenes como sujetos pensantes $\mathrm{y}$ actuantes, poseedores de una sensibilidad propia y de una serie de competencias y habilidades que muchas veces superan con creces las de los adultos y que, antes de comprometerse en o con algo reflexionan sobre la "utilidad" y el "placer" que encontrarán en ello, de modo que dicho compromiso podrá aportarles, tanto en su desarrollo personal como profesional.

Para Delgado y Arias (2008), la construcción de marcos de acción colectiva donde se exponen los horizontes de sentido y las intenciones de las prácticas de los jóvenes permite vislumbrar uno de sus elementos constitutivos: la explicitación de los problemas y conflictos sociales relevantes para ellos. Esto nos muestra su capacidad de reflexividad $y$ de sensibilidad moral para producir controversia respecto a situaciones que los afectan, y cuya legitimidad y sentido normativo se daban por incuestionables. Delgado y Arias destacan que los procesos de crítica y autocrítica permanente con que los colectivos juveniles desarrollan su accionar se expresa en la definición de esquemas de lectura para interpretar los acontecimientos que agravian su dignidad, y que por su parte es percibida no simplemente como una desgracia sino como una injusticia, estableciendo paulatinamente posturas de interpretación compartidas desde las cuales justifican y legitiman sus acciones colectivas. Otro aspecto a resaltar es que esta capacidad para establecer juicios y orientar sus decisiones prácticas para promover cambios sociales en sus contextos más inmediatos, proviene de sentimientos morales que sustentan la celebración de vínculos de solidaridad, desde los cuales construyen un sentido del nosotros como integrantes de una comunidad ética y política a la cual se vinculan a partir de iniciativas que demandan inclusión, igualdad jurídica y reconocimiento recíproco, entre otros aspectos.

Con el objetivo de indagar si hay algún tipo de relación entre la vinculación de los jóvenes a organizaciones comunitarias y sus percepciones sobre las problemáticas del entorno, se indagó acerca de su posición ante las problemáticas juveniles. Sus respuestas se especifican en la Tabla 7:

E1 55,4 \% afirmó que siempre reflexiona sobre la falta de oportunidades de educación y trabajo en los jóvenes, mientras que el 50,5 \% y el 50,1 $\%$ dijo, de manera respectiva, que les preocupan la drogadicción y el embarazo adolescente, y un último $34,49 \%$ dijo preocuparse siempre por la prostitución.

Otro conjunto de datos que proporcionó información sobre los problemas sociales que afectan a la juventud, se evidenció mediante unas notas anónimas y otras firmadas que algunos estudiantes y padres de familia dejan 
Tabla 7. Reflexión sobre problemas juveniles

\begin{tabular}{|l|c|c|c|c|c|}
\hline $\begin{array}{l}\text { 32. SS15. ¿Qué tanto te preocupas 0 reflexionas sobre los } \\
\text { siguientes problemas juveniles? }\end{array}$ & Nunca & Algunas veces & Casi siempre & Siempre & No Contestó \\
\hline Prostitución & 19,86 & 13,59 & 27,87 & 34,49 & 4,18 \\
\hline Drogadicción & 15,68 & 7,67 & 20,91 & 50,52 & 5,23 \\
\hline Embarazo adolescente & 14,29 & 9,76 & 21,95 & 50,17 & 3,83 \\
\hline Injusticias & 9,41 & 7,67 & 27,87 & 48,78 & 6,27 \\
\hline Falta de oportunidades de educación y trabajo & 9,06 & 7,67 & 23,00 & 55,40 & 4,88 \\
\hline
\end{tabular}

Fuente: Elaboración propia

debajo de la puerta de la oficina del rector de la institución educativa de Cartago (se consultaron 30). En dichas notas se aclaran situaciones disciplinarias, se señalan nombres de estudiantes que habrían transgredido las normas, se denuncian acciones de docentes y se reclama igualdad en la aplicación de las sanciones. Llamó mucho la atención la reiterada aparición de denuncias y peticiones de ayuda relacionadas con el tema de la drogadicción. Aunque la drogadicción es un problema que se presenta en todos los estratos de la sociedad, en los colegios el consumo es asociado con estudiantes con problemas psicoafectivos, sociales y económicos para los que las estrategias pedagógicas de la institución resultan insuficientes. Al respecto, los propios educadores manifiestan que carecen de herramientas para solucionar este problema y que, además de una serie de contenidos y metodologías, necesitan obtener más conocimiento y desarrollar competencias para abordarlo. A continuación se presenta el contenido de algunas notas dejadas por los estudiantes y padres al rector:
Tabla 8. Notas debajo de la puerta de institución educativa solicitando ayuda para tratar temas

\section{de drogadicción en los alumnos}

Nota 1: Rector, soy estudiante del grado $9^{\circ} 1$, me acerco a usted para pedirle por dos estudiantes de grupo, ellos están empezando a consumir vicio los vi el martes en el coliseo

Nota 2: Señor Rector soy la madre y acudiente de un estudiante del grado $8^{\circ} 3$, le escribo para solicitarle que no saquen a mi hijo de colegio por el rumor que hay de consumo de drogas, yo como madre me comprometo a estar más pendiente de él y vigilar sus compañías

Nota 3: Yo soy estudiante del grado $8^{\circ} 2$ quiero aclarar lo que pasó en el paseo. Cuando nos desplazábamos en el bus, un joven se puso a mostrar marihuana en un papel de cuaderno y luego lo guardó en su bolso. Al rato sacaron cigarrillos mentolados y me dieron a mí, y mi compañero, nosotros fumamos cigarrillos mentolados, pero no marihuana como están diciendo, nosotros nos dedicamos fue a divertirnos, son testigas unas compañeras con las que nosotros estuvimos charlando. Colabóreme, yo quiero salir adelante, mi error fue no haberme quitado de allí, cuando el compañero mostraba la marihuana

Nota 4: Me permito informarle que usted dijo que le ayudáramos con la limpieza del colegio. Le voy a dar los nombres de los muchachos que están metiendo solución en el colegio pero más que todo en el coliseo, yo no quiero que mi hermana que está en primaria siga los mismos ejemplos cuando pase a bachillerato, por este motivo le voy a dar los nombres de ellos

Nota 5: Cuatro compañeros de grado $6^{\circ} 2$ vimos a dos compañeros fumando cigarrillo en la parte de atrás, y en el descanso un muchacho de la calle les pasaba una bolsita con un contenido, nosotros le dijimos a una profesora pero esperamos que usted también haga algo 
Los testimonios de los alumnos reafirman que el consumo y expendio de drogas son una problemática patente en los establecimientos de educación secundaria, donde diferentes espacios $\mathrm{y}$ actividades de las instituciones son utilizados por alumnos y por personas externas para inducir a los jóvenes al consumo. Inquieta mucho que, ante la problemática, las autoridades educativas hayan solicitado a los alumnos colaborar "con la limpieza del colegio", es decir, delatar a los consumidores y expendedores para sacarlos de la institución, incurriendo claramente en una práctica de eliminación del problema por la vía del retiro de los estudiantes del servicio educativo y reproduciendo prácticas de segregación y marginación social, que han sido utilizadas fuera de la escuela y que han conducido al uso de la violencia contra drogadictos. Lo anterior preocupa porque al igual que ha pasado fuera de la escuela, la solución al consumo de drogas vía "limpieza social" profundiza los problemas sociales que desencadena el consumo e impide que la drogadicción reciba el tratamiento de problema de salud pública que merece. En el caso de los estudiantes, si estos son retirados del colegio se pierde la oportunidad de recibir los apoyos médicos, afectivos y sicológicos que garanticen el tratamiento integral de su situación.

En este contexto, conviene citar a Kessler (2002), quien enfatiza que la vida exterior ocupa un lugar mayor de lo que se cree en la escuela, los conflictos sociales se instalan en la clase, en un marco en que la sociedad en general, y las escuelas en particular, son cada vez más heterogéneas entre sí en cuanto a públicos y recursos. La subjetividad se construye de una manera muy tamizada y por lo que podrían llamarse experiencias de clase particulares, sin las tendencias compensadoras de un sistema educativo más homogéneo.

\section{Discusión}

La socialización fue abordada como una de las lógicas de acción (la Lógica de la Integración) en la propuesta teórica de Dubet (2010). Este autor entiende la socialización como una experiencia social, orientada por una pluralidad de roles y dimensiones que interactúan, sin que un principio central pueda organizarla. Más en concreto, la socialización se asume como el ámbito y el espacio en el cual se produce la construcción de subjetividad política, específicamente en la construcción de actitudes y concepciones políticas.

En este trabajo se partió de dos cuestiones: por un lado, una modificación significativa en el equilibrio de poder entre las generaciones y la cultura propia de los jóvenes estudiantes de hoy; y por el otro, la convivencia de los estudiantes en espacios de socialización en transformación como la familia y la escuela.

La postura de Dubet (2010) y los datos empíricos recogidos para la investigación permitieron confirmar que la socialización de los estudiantes se realiza en dos ámbitos, el escolar y el externo a la escuela. En estos dos ámbitos se entablan relaciones afectivas y otras 
de simple convivencia con la familia, los pares, los docentes y los vecinos; también se identificaron percepciones e influencias de problemáticas sociales de sus entornos y de los consumos culturales de la actualidad.

Los trabajos empíricos de Delgado y Arias (2008) plantean que la acción colectiva agenciada por los jóvenes no solo refleja un juicio intelectual y cognitivo sobre lo que es equitativo o no, sino que también encierra una alta carga emotiva y afectiva relacionada con la indignación, el resentimiento, el desprecio social, el abandono y la solidaridad como factores motivacionales presentes en sus procesos de organización, participación y movilización social. En consecuencia, estos autores resaltan los marcos de justificación ético-política presentes en las diversas narraciones y testimonios de los jóvenes, destacando tres grandes referentes en sus justificaciones: los sentimientos morales, los derechos humanos y el reconocimiento recíproco.

Velásquez, Martínez y Cumsille (2004, siguiendo a Gilligan, 1985) introducen un punto interesante a la discusión cuando subrayan que es posible identificar en los resultados dos tipos de participación, diferentes entre sí pero correlacionados: la participación prosocial y la participación activista, asociadas a la socialización femenina y masculina respectivamente. Desde la perspectiva de género, estos autores precisan que la participación prosocial responde a la ética de cuidado y la participación activista, a la ética del derecho.
Este hallazgo es el elemento inicial para continuar con las posteriores hipótesis de investigación, ya que si existen estos dos tipos de participación, es posible entonces plantear que eventualmente no existen diferencias en la cantidad de participación de hombres y mujeres, sino que su participación es diferente en relación al tipo de actividad elegida. En palabras de Velásquez, Martínez y Cumsille (2004), el problema no es que las mujeres participen menos, sino que la forma en que se ha evaluado la participación podría no ser sensible a las diferencias de género, ya que las mujeres lo hacen con una orientación diferente y en actividades distintas.

La investigación de Muñoz (2003) concluyó, por otra parte, que la falta de participación de los jóvenes se relaciona con la debilidad de las políticas públicas; desde su punto de vista, las políticas de juventud florecieron silvestres, se improvisaron, no tuvieron un norte, como tampoco metas productivas o bases que las sustentaran. Además, carecieron de interlocutores válidos, de fundamento investigativo suficiente y de un debate abierto y transparente. Para este autor, las políticas de juventud fueron una inmensa fachada, detrás de la cual no había contenidos estructurados. En consecuencia, su ejecución ha sido errática, producto de una desafortunada gestión que no dimensiona las actuaciones concretas que podrían transformar a cada país, si hubieran comprometido acciones con estos actores estratégicos.

En la misma perspectiva de Muñoz, Sandoval 
(2000) considera que las razones de la baja participación política de los jóvenes, se debe a razones estructurales, a una crisis de la cual son sujetos, dado que las organizaciones sociales no poseen el sentido pertinente para que estos se organicen y participen de ellas. Recalca que las formas de asociación destinadas a la población juvenil, si es que existen, más que fomentar su participación, los inhiben y los obligan a configurar nuevas formas asociativas, transgrediendo la normativa social a la cual deben adecuarse. Para Sandoval (2000), no es congruente que se exija a los jóvenes que se vinculen a organizaciones sociales si no existen el espacio ni el sentido para ser partícipes de una sociedad que insiste en comprender a la juventud actual desde los parámetros de las décadas de los sesenta y setenta. En sus propias palabras: "la voluntad de participación ciudadana en el mundo juvenil tiene como base la confianza en las instituciones, cuestión que está lejos de darse, así como también la conciencia juvenil de influir y ser escuchados por las mismas. Al no presentarse estas condiciones mínimas, resulta imposible demandar a la población la participación necesaria para que vuelvan a ser considerados como el porvenir del mundo y no como amenaza y población al margen de la sociedad" (Sandoval, 2000, p.151).

Contrario a algunos imaginarios desde los cuales se percibe que la escuela ha perdido su función de agente de socialización, algunos autores coinciden en que esta es el escenario ideal para formar a niños y jóvenes comprometidos con sus comunidades. El trabajo de
Loreto, Silva, Morandé y Canales (2010), por ejemplo, problematiza sobre el papel potenciador que tendría la escuela para animar en los jóvenes la vinculación con organizaciones comunitarias. Estos autores argumentan que algunos programas promovidos desde la escuela se anclan en actividades establecidas en la institución, como clases de historia o de orientación. Según este grupo de investigadores es necesario que se resignifiquen acciones que desde la escuela promuevan la vinculación de jóvenes a las dinámicas comunitarias para aprovechar de manera más efectiva las oportunidades que los programas de formación ciudadana ofrezcan en el sentido de establecer vínculos sociales y de conectarse con otros. De otro lado, en la medida en que estos vínculos validen las capacidades de las personas, se desarrollará un sentido de identidad colectiva que promueva los valores y actitudes de compromiso con los otros y con el bienestar común.

En esta perspectiva, Torres (2001) considera que, para convertirse en instrumento de desarrollo, empoderamiento y equidad social, la participación debe involucrar a todos los actores, diferenciando y a la vez sincronizando sus roles, así como darse en los diversos ámbitos y dimensiones de lo educativo: desde el aula de clase hasta la política educativa, en la educación escolar y también en la extra-escolar, en los aspectos administrativos y en los relacionados con la enseñanza y el aprendizaje, a nivel local y a nivel nacional y global. Según este autor, esto implica el estudio, la definición y puesta 
en marcha de una estrategia de participación social imbricada en la propia política educativa y acordada participativamente, a fin de delimitar con claridad roles y responsabilidades de cada uno de los actores y asegurar las condiciones y los mecanismos para hacer efectiva dicha participación.

Las ideas planteadas en este artículo sugieren que en materia de vinculación de los jóvenes a dinámicas comunitarias, tenemos más retos que resultados. Krauskopf (2000) propone algunos de ellos: problematizar los procesos de construcción de conocimientos sobre la adolescencia y juventud para reducir las barreras existentes en el fomento del desarrollo juvenil, reconocer los derechos y capacidades de las juventudes, no bloquear sus aportes. Concluye que la diversificación programática, con participación efectiva de jóvenes y comunidades, proyección y vinculación a las políticas, articuladas entre sí y enriquecidas con los nuevos paradigmas, posibilita procesos acordes con las necesidades de desarrollo actual de las juventudes.

\section{Conclusiones}

En cuanto a su vínculo con las actividades comunitarias, los alumnos objeto de este trabajo destacan su participación en celebraciones y eventualmente en acciones para recolectar dinero para todo el vecindario o para alguno de sus miembros. Afirman, también, que dividen su tiempo entre las actividades que los divierten y la asistencia al colegio. Entre las personas que identifican como influyentes en sus decisiones figuran, en su orden, la madre, el padre, los amigos, los profesores y la pareja. Por otra parte, dicen tener más afinidades con personas de la misma edad y los mismos gustos, destacando que no les interesa tener ningún tipo de relación y cercanía con quienes consideran diferentes a ellos. La gran mayoría vive con los padres o con uno de ellos, pero también hay quienes viven con otros familiares, como tíos, primos y abuelos.

Sobre su participación en agrupaciones distintas a la escuela, se destaca la vinculación con actividades deportivas; en tanto que la relación con los grupos de pares se establece principalmente en el colegio, las esquinas de las cuadras y los espacios virtuales; en consecuencia, los objetos más preciados para los estudiantes son el televisor, el celular, el computador, los videojuegos y el equipo de sonido. En un contexto como el que nos ilustran Sánchez y Robles (2016) en el que la complejidad en las relaciones interpersonales en la infancia y adolescencia tiene un nuevo añadido: el mundo digital, que produce riesgos y potencialidades, un fuerte vínculo de los jóvenes con la vida comunitaria, puede constituirse en un medio de protección frente a dichos riesgos, las actividades comunitarias pueden significar para ellos la apertura a ambientes seguros, donde además de desplegar sus capacidades, reciban acompañamiento de sus pares y de los adultos.

El estudio evidenció una relativa baja participación de los estudiantes en las actividades y grupos de sus comunidades. Este hecho preocupa por el gran valor que pueden tener 
las organizaciones comunitarias en sociedades como la nuestra, tales como gestionar recursos y actuar como veedoras y fiscalizadoras de irregularidades en su uso y ejecución. No obstante, esta posibilidad es lejana si, como expresan los datos, la principal actividad en la que los jóvenes se acercan a la comunidad consiste en recoger dinero para celebraciones, y muy pocos se vinculan a actividades que les permitan discutir y proponer soluciones a los problemas comunitarios.

En otro sentido, aunque relacionado con todo lo anterior, se necesitan estudios que indaguen sobre la efectividad de las políticas de juventud que se han planeado e implementado en los últimos años. Estos estudios pueden dar cuenta sobre el impacto de las instancias gubernamentales que se han creado tanto a nivel del estado central como regional y local, así como las acciones que se han puesto en marcha.

También es necesario indagar al interior de la escuela sobre el papel que esta cumple en la formación de niños y jóvenes que estén dispuestos a integrarse a proyectos comunitarios. Dicha indagación debe abarcar una lectura de los proyectos educativos institucionales, el currículo y los discursos y prácticas que circulan entre los estudiantes, los directivos y los docentes sobre este tema.

Finalmente, es necesario conocer la visión de las organizaciones comunitarias. Para ello, se precisan investigaciones sobre las motivaciones que acercan o alejan a los jóvenes de sus organizaciones, y las acciones que desde sus puntos de vista deben adelantarse para que ellos se interesen y animen a participar en dichas agrupaciones.

\section{Referencias}

Alvarado, S. \& Ospina, H. F. (2007). Las concepciones de equidad y justicia en niños y niñas. Desafíos en los procesos de configuración de subjetividad política. En E. Vasco, S. Alvarado, C. Echavarría \& P. Botero (Ed.), Justicia moral y subjetividad política en niños, niñas y jóvenes (pp.81100). Manizales, Colombia: Centro de Estudios Avanzados en Niñez y Juventud. CINDE.

Bandit, R. (1999). Participación social y política de los jóvenes en países de la Unión Europea. Ponencia presentada durante la Reunión del Grupo de Trabajo “Juventud". CLACSO.

Castro, G. \& Molinari, V. (2009). Eje prácticas culturales, estilos, consumos y estéticas. En M. Chaves (Ed.), Estudios sobre juventudes en Argentina I. Hacia un estado del arte. (pp. 269-275). Buenos Aires, Argentina: Universidad Nacional de La Plata.

De la Torre, E. \& Navarro, R. (1990). Metodología de la investigación. Bibliografía, archivistica y documental. México: Editorial McGraw-Hill.

Delgado, R. (2005). Análisis de los marcos de acción colectiva en organizaciones sociales de mujeres, jóvenes y trabajadores. Trabajo de grado para optar por 
el título de Doctor en Ciencias Sociales, Niñez y Juventud. Universidad de Manizales - CINDE.

Delgado, R. \& Arias, J. (2008). La acción colectiva de los jóvenes y la construcción de ciudadanía. Revista Argentina de Sociología, 6(11), 272-296.

Dubet, F. (2010). Sociología de la experiencia. Madrid, España: Centro de Estudios Sociológicos. Editorial Universidad Complutense de Madrid.

Garcés, A. (2010). De organizaciones a colectivos juveniles panorama de la participación política juvenil. Revista Última Década, (32), 61-83.

Hernández, R., Fernández, C. \& Baptista, P. (2003). Metodología de la investigación. Los procesos mixtos o multimodales. México: McGraw-Hill Interamericana.

Kessler, G. (2002). La experiencia escolar fragmentada. Buenos Aires: IIPE. UNESCO.

Krauskopf, D. (2000). Dimensiones críticas en la participación social de las juventudes. En S. Balardini (Comp.), La participación social y política de los jóvenes en el horizonte del nuevo siglo. Buenos Aires: CLACSO.

Lechner, N. (1999). Las condiciones sociopolíticas de la ciudadanía. Citada por Alvarado, S. \& Ospina, H. F. (2007). Las concepciones de equidad y justicia en niños y niñas. Desafíos en los procesos de configuración de subjetividad política. En E. Vasco, S. Alvarado, C. Echavarría \& P.
Botero (Ed.), Justicia moral y subjetividad politica en niños, niñas y jóvenes (pp.81100). Manizales, Colombia: Centro de Estudios Avanzados en Niñez y Juventud. CINDE.

Loreto, M., Silva, C., Morandé, M. \& Canales,L. (2010). Los jóvenes ciudadanos: Reflexiones para una política de formación ciudadana juvenil. Revista Última Década, (32), 105-118.

Muñoz, G. (2003). Temas y problemas de los jóvenes colombianos al comenzar el siglo XXI. Revista Latinoamericana de Ciencias Sociales, Niñez y Juventud, 1(1), 145-180.

Palacios, N. (2008). Disciplina, norma y democracia sus concepciones y funcionamientos en la cotidianidad de la escuela. (Trabajo de grado para optar por el título de Magíster en Sociología). Facultad de Ciencias Sociales y Económicas, Universidad del Valle.

Sánchez, T. \& Robles, M. (2016). Riesgos y potencialidades de la era digital para la infancia y la adolescencia. Revista Educación y Humanismo, 18(31), 186-204.

Sandoval, M. (2000). La participación social y política de los jóvenes en el horizonte del nuevo siglo. En S. Balardini (Comp.), La participación social y política de los jóvenes en el horizonte del nuevo siglo. Buenos Aires: CLACSO.

Tanaka, N. (1995). Jóvenes: actores sociales y cambio generacional. De la acción 
colectiva al protagonismo individual. Economía, sociedad y politica. Lima: IEP.

Torres, R. (2001). Participación ciudadana $y$ educación. Una mirada amplia $y$ 20 experiencias en América Latina. Documento encargado por la Unidad de Desarrollo Social y Educación (UDSE) de la OEA para su presentación en la Segunda Reunión de Ministros de Educación del Consejo Interamericano para el Desarrollo Integral -CIDI. Punta del Este, Uruguay, 24-25 septiembre.

Vasco, E., Quintero, M. \& Echavarría, C. (2007).
Algunas concepciones de justicia de un grupo de estudiantes universitarios de dos ciudades del país. En E. Vasco, S. Alvarado, C. Echavarría \& P. Botero (Ed.), Justicia moral y subjetividad política en niños, niñas y jóvenes (pp.81-100). Manizales, Colombia: Centro de Estudios Avanzados en Niñez y Juventud. CINDE. Velásquez, E., Martínez, L. \& Cumsille, P. (2004). Expectativas de autoeficacia y actitud prosocial asociadas a participación ciudadana en jóvenes. Revista PSYKHE, 13(2), 85-98. 